\title{
ANÁLISIS DEL ENTORNO FUNCIONAL DEL SECTOR EMPRESARIAL EN EL DEPARTAMENTO DEL CESAR
}

\section{FUNCTIONAL ANALYSIS OF THE BUSINESS SECTOR ENVIRONMENT IN THE DEPARTMENT OF CESAR}

\author{
Jairo Enrique Polo Martínez
}

Forma de citar: POLO-MARTÍNEZ Jairo Enrique. Análisis del entorno funcional del sector empresarial en el departamento del Cesar. Respuestas. 2014; 19(1):50-65.

Recibido:

Noviembre 6 de 2013

Aceptado:

Febrero 27 de 2014

\section{RESUMEN}

Antecedentes: El entorno funcional de la empresa en el departamento del Cesar es un escenario innato para los entes que planean el futuro socioeconómico de la región. Dicho entorno está compuesto por una enorme cantidad de agentes, factores y relaciones que influyen directa o potencialmente sobre la empresa condicionando el éxito competitivo de una manera tan relevante que es una de las razones fundamentales que explica el fracaso empresarial. La actual investigación toma como base el "Entorno funcional de la Provincia en Sevilla", donde se definen las variables y sus relaciones según la lógica del sistema completo de los determinantes de la ventaja de Michael Porter. Objetivo: el presente estudio busca analizar el entorno funcional de la empresa agroindustrial en el departamento del Cesar, a través de la aplicación de modelos estadísticos de correlación, permitiendo obtener una aproximación a la definición de su tipología estructural y el nivel de calidad empresarial de las mismas. Método: Las variables seleccionadas fueron esquematizadas en la encuesta aplicada a 23 empresas agroindustriales representativas del Cesar. La investigación plantean 16 hipótesis con el fin de definir relaciones sobre: tamaño, antigüedad, calidad, innovación, relación comercial con administraciones públicas, necesidad de financiación, hostilidad del entorno, control, conciencia social empresarial, entre otras. Las relaciones se validan a través de la técnica del coeficiente de correlación de Pearson y Covarianza. Los datos resultantes, son sometidos a un análisis y comparación con el proyecto de Sevilla (España). Posteriormente, se tabula la información y se establecen los perfiles empresariales del sub-sector agroindustrial en el departamento del Cesar. Resultado: De los resultados obtenidos, tres (3) de las hipótesis son validas y trece (13) se descartan, a diferencia de la investigación en Sevilla donde existen mayor relación entre las variables estudiadas (9 validas), debido a las diferencias microeconómicas, territoriales y cultura empresarial. De lo anterior, se afirma que entre las empresas estudiadas existe una gran relación entre el tamaño de las empresas con las que se coopera y la ubicación física de las empresas con las que se establece algún acuerdo de cooperación. Además, el tamaño de las empresas se relaciona 
significativamente con la certificación de los sistemas de calidad, es decir, las empresas con mayor tamaño son las que tienden certificar sus sistemas de gestión de la calidad. La última relación validada en las hipótesis, establece que las empresas con un control formalizado, son las que promocionan a sus directivos según su experiencia y capacitación profesional, en detrimento de la creatividad. Conclusiones: En cuanto a la orientación al cliente, se afirma un alto potencial innovador de las empresas agroindustriales en el departamento del Cesar, pero con bajo niveles de sistemas de gestión de la calidad certificados, aunque ambas variables se enfocan a la satisfacción del cliente, los productos no sobrepasan los clientes de la región y el poder de decisión es significativamente bajo. Finalmente, se pudo determinar dos principales problemas: primeramente la materia prima, que en general, encierra aspectos de calidad, transporte y disponibilidad; en segunda instancia la Maquinaria, asociado a necesidades de nuevas piezas que en el mercado Colombiano no se producen o comercializan, además de los requerimientos de tecnología especializada.

Palabras claves: Entorno Funcional, Agroindustria, Perfil Empresarial y Correlación.

\section{ABSTRACT}

Background: The functional environment of the company in the department of Cesar is an innate scenario planning authorities for the socioeconomic future of the region. This environment consists of a huge number of agents, factors and relationships that influence directly or potentially conditional on the company's competitive success of such a substantial way that is one of the main reasons explaining business failure. The current research builds on the "functional environment in Seville Province", where the variables and their relationships are defined according to the logic of the entire system of the determinants of competitive advantage Michael Porter. Objective: The present study aims to analyze the functional environment of agribusiness in the department of Cesar, through the application of statistical correlation models, allowing an approximation to the definition of the structural type and level of quality business same. Method: The selected variables were sketched in the survey of 23 representative Cesar agribusinesses. 16 The research hypotheses posed in order to define relationships on: size, age, quality, innovation, business with government, borrowing, hostile environments, control, corporate social awareness, among others. The relationships are validated through the technique of Pearson correlation coefficient and covariance. The resulting data are subjected to analysis and comparison with the draft Seville (Spain). Then the information is tabulated and business profiles agribusiness sub-sector in the department of Cesar down. Results: From the results obtained, three (3) of the assumptions are valid and thirteen (13) are discarded, unlike research in Seville where most relationships among the studied variables (9 valid) are due to microeconomic differences territorial and corporate culture. From the foregoing, it is claimed between the companies studied 
a relationship between the size of the companies with which it cooperates and the physical location of the companies with which a cooperation agreement is established. In addition, the size of firms is significantly related to the certification of quality systems, that is, firms with larger are the most likely to certify their management systems quality. The last relationship validated hypothesis, states that companies with a formalized control are touting their managers according to their experience and training, at the expense of creativity. Conclusions: In terms of customer orientation, a high innovative potential of agro-industrial companies in the department of Cesar, but low levels of management systems certified Quality states, although both variables are focused on customer satisfaction, products do not exceed clients in the region and the power of decision is significantly low. Finally, it was determined two main problems: first the raw material, which generally includes aspects of quality, transport and availability; Machinery on appeal, needs associated with new parts in the Colombian market does not produce or market, in addition to the requirements of specialized technology.

Keywords: Functional Environment, Agribusiness, Business Profiles and Variable's Correlation.

\section{INTRODUCCIÓN}

$\square^{1}$

presente proyecto es apoyado por el programa jóvenes investigadores innovadores del Departamento Administrativo de Ciencia, Tecnología e Innovación (COLCIENCIAS).

El Departamento del Cesar (Colombia), se caracteriza económicamente por dos actividades principales, la minería y la agricultura [1]. Dada la relevancia histórica a nivel social, la vocación agropecuaria de los municipios de la región cesarense y la evidente disminución paulatina y constante del PIB del sector agrícola del departamento del Cesar desde los inicios de los años noventa representada en un 20\% aproximadamente. La investigación busca analizar el entorno funcional de la empresa agroindustrial en el departamento del Cesar, a través de la aplicación de modelos estadísticos de correlación que permita una aproximación a la definición de su tipología estructural y el nivel de calidad empresarial y organizacional [2].

Inicialmente se estructura un diagnostico a partir de encuestas aplicadas, posteriormente se validan las hipótesis establecidas. Finalmente, se proponen perfiles empresariales teniendo en cuenta la integración de las variables estudiadas.

El entorno funcional de la empresa en el departamento del Cesar es un escenario innato para los entes que planean el futuro socioeconómico de la región; dicho entorno está compuesto por una enorme cantidad de agentes, factores y relaciones que influyen directa o potencialmente sobre la empresa, condicionando el éxito competitivo de una manera tan relevante que es una de las razones fundamentales que explica el fracaso empresarial [3]. El entorno funcional en de las empresas este territorio es complejo y 
los pocos estudios formales realizados hasta la fecha han abordado situaciones muy particulares relacionadas en su mayoría con la minería [4] el sector agropecuario [5] [6] o desde la óptica de la evolución histórica de un sector de la economía regional [7] y sus implicaciones sociológicas [8].

El presente documento se estructura inicialmente enunciando los materiales y métodos considerados para el desarrollo de la investigación, posteriormente se presentan los resultados obtenidos durante el cumplimiento de los objetivos, seguido de las conclusiones que permitan discutir y enunciar las ideas principales a considerar por los empresarios, administraciones publicas y sociedad en general. Además, los investigadores expresan los agradecimientos a las entidades que fueron significativas y apoyaron el proceso investigador, y finalmente, las literaturas consultadas.

\section{MATERIALES Y MÉTODOS}

\section{DISEÑO DEL ESTUDIO}

La investigación tiene una forma aplicada, la cual es el estudio y aplicación de la investigación a problemas concretos, en circunstancias y características concretas, es decir esta forma de investigación se dirige a su aplicación inmediata y no al desarrollo de teorías [9]. La investigación es de tipo exploratoria, en su primera fase, debido a que el fenómeno no ha sido estudiado en el Departamento, específicamente en el sector Agroindustrial. Posteriormente, se considera este estudio como una investigación de tipo descriptivo, donde se toman datos obtenidos de la realidad, se describen a través de análisis de los factores principales que hacen parte del entorno funcional de las empresas agroindustriales del departamento del Cesar [10] [11]. Por otra parte, el análisis efectuado en la presente investigación determina un enfoque cuantitativo [10].
Las variables esenciales del sistema que se estudio son elegidas en el estudio original por su interés para la descripción del entorno empresarial basados en la lógica del modelo de los determinantes de las ventajas de Michael Porter [12], y conocidos los excelentes resultados del estudio realizado en la Universidad de Sevilla, se decide utilizar variables contextualizadas con el entorno Cesarense.

La cantidad de variables necesarias debe ser numerosa y con mayor carga cualitativa, para plantear adecuadamente modelos de realidades complejas [13]. Para el presente proyecto se considera seleccionar 15 variables. Esta cifra no es extraña debido a que estudios anteriores como el de Lober y Bailey, donde se utilizaron 18 cuestiones en escalas tipo Likert para medir las 15 variables independientes en el que se plantean 15 hipótesis. Este número de variables es razonable y suficiente para modelar correctamente el entorno, permitiendo estudiar abundantes relaciones potenciales entre las variables [14].

\section{Variables a estudiar}

- Datos Genéricos de Identificación: antigüedad o edad y tamaño de las empresas.

- Orientación hacia la Competencia: tipo de innovación, dinamismo de la competencia y compromiso con la calidad

- Orientación hacia los Clientes: grado de concentración de las ventas

- Orientación hacia el Talento Humano: dificultad en la selección del talento humano; experiencia y capacitación profesional en la promoción de los directivos y grado de control sobre la plantilla PN 0122-820X P: $50-65$

Orientación hacia la Asociatividad: tamaño de las empresas con las que se coopera y localización geográfica de las empresas colaboradoras 
No. 1
- Orientación hacia la Esfera Financiera: necesidad de financiación ajena

- Orientación hacia las Administraciones Públicas: facturación al sector público y sentimiento de respaldo institucional

- Orientación hacia la Sociedad: contribución a la creación de riqueza nacional como objetivo de la empresa

\section{HIPÓTESIS OPERATIVAS}

- Hipótesis 1: En el entorno del Departamento del Cesar existe una gran relación entre el tamaño de las empresas con las que se coopera y la ubicación física de las empresas con las que se establece algún acuerdo de cooperación.

- Hipótesis 2: En el entorno Cesarense existe relación entre la proximidad física de las empresas que cooperan y el desarrollo de innovaciones, de tal forma que la internacionalización en la realización de acuerdos favorece el desarrollo de innovaciones.

- Hipótesis 3: El tamaño de las empresas del departamento del Cesar aumenta con la edad de las empresas.

- Hipótesis 4: El tamaño de las empresas del departamento del Cesar se relaciona significativamente con la certificación de los sistemas de calidad.

- Hipótesis 5: El tamaño de las empresas del departamento del Cesar no es una variable relacionada con la innovación.

- Hipótesis 6: Las empresas agroindustriales más innovadores son las que más venden a las administraciones públicas.

- Hipótesis 7: Las empresas con mayor calidad son las que más venden a las administraciones públicas.

- Hipótesis 8: En el entorno del
Departamento del Cesar las empresas con más calidad son las más innovadoras.

- Hipótesis 9: La necesidad de financiación de la empresa aumenta a medida que aumenta la hostilidad del entorno.

- Hipótesis 10: La hostilidad del entorno provoca la concentración de los clientes.

- Hipótesis 11: La hostilidad o rivalidad en el entorno del Departamento del Cesar no repercute directamente en un aumento de la innovación.

- Hipótesis 12: Las organizaciones con más calidad son las que promocionan a sus directivos según su experiencia y capacitación profesional, en detrimento de la creatividad.

- Hipótesis 13: Las organizaciones con un control formalizado son las que promocionan a sus directivos según su experiencia y capacitación profesional, en detrimento de la creatividad.

- Hipótesis 14: Las organizaciones más concientizadas socialmente son las que tienen menos problemas para reclutar personal.

- Hipótesis 15: Las organizaciones con más calidad son las tienen más problemas a la hora de reclutar el personal.

- Hipótesis 16: Las organizaciones que mantienen relaciones comerciales con las administraciones públicas son las que perciben en mayor medida la existencia de apoyo de las administraciones públicas.

\section{DEFINICIÓN DE SUJETOS DE ESTUDIO:}

2.3.1 Población: La población es el conjunto de todos los elementos que presentan una característica determinada o que 
corresponden a una misma definición y a quienes se les estudiaran sus características y relaciones [15]. En este sentido, la población para este proyecto estará conformada por las 23 empresas agroindustriales legalmente constituidas, representativas de los municipios del Departamento del Cesar, pertenecientes a los subsectores: procesamiento de fruta de Palma, Lácteos y Cárnicos; Frutas y hortalizas; Granos y cereales [16] [17] [18]

Es de resaltar que la definición de la población fue un proceso que se alimento con el desarrollo de la investigación ya que las fuentes consultadas la información presentaba empresas desactualizadas o con clasificación innecesarias para el proyecto.

Unidad de Análisis: La unidad de análisis está conformada por los elementos de donde se va a obtener la información para ser analizada y en este caso se trabajara con base en está conformada por las empresas agroindustriales legalmente constituidas, representativas de los municipios del Departamento del Cesar, pertenecientes a los subsectores: procesamiento de fruta de Palma, Lácteos y Cárnicos; Frutas y hortalizas; Granos y cereales [19].

Unidad de Muestreo: La unidad de muestreo es una parte de los elementos de la población. [15]. Como se ha indicado, la población de estudio está conformada por veintitrés (23) Empresas agroindustriales del departamento del Cesar; dadas las características de esta población, se tomaran como unidades de muestreo todos los sujetos que la integran, haciendo que la población y la muestra coincidan.

Técnica de Muestreo: En esta investigación se aplicará el muestreo no aleatorio intencional, el cual constituye en seleccionar los elementos que a juicio del investigador son los más representativos [19]. Las unidades muéstrales serán elegidas por el investigador de manera arbitraria, designando a cada unidad según las características que sean de mayor relevancia para el investigador, por lo tanto se empleara el conocimiento y la opinión personal para identificar aquellos elementos que deben ser incluidos en la muestra.

\section{PROCEDIMIENTO}

Las diferentes fases del método son las siguientes:

- Determinación del listado de las var ables.

- Selección de las empresas: Búsqueda en base de datos ( Cámara de Comercio, FENALCO, entre otras)

- Búsqueda de datos de calidad por medio de aplicación de encuesta y entrevista (asegurar que quienes los proporcionan tengan responsabilidades ejecutivas suficientes y estén cualificados para manejar la información)

- Descripción de relaciones entre variables utilizando el software IBM SPSS Statistics

- Identificación de los tipos de estructura empresarial.

- Determinación de la calidad empresarial.

- Proposición de perfiles.

\section{RESULTADOS}

\section{VALIDACIÓN DE HIPÓTESIS}

Para la validación de las hipótesis, se utiliza el software IBM SPSS STATISTICS versión 22, aplicando el coeficiente de Pearson y análisis de covarianza, porque este permite identificar la correlación lineal entre dos variables, para la validación se tuvo en cuenta el coeficiente de Pearson de 0 hasta 0,4 o - 0,4 como invalida y desde 0,401 hasta 1 o $-0,401$ hasta -1 , como válida. Además, apoyado de la investigación
Enero - Junio 2014

ISSN 0122-820X

PP: $50-65$ 
se apoya en la covarianza corroborando si la correlación lineal es directa (positiva) o inversa (negativa) [20]

Inicialmente la investigación plantea utilizar el mapa bidimensional de relaciones utilizado en la investigación de Sevilla [19], este es de carácter empírico, sumado a la falta de información para la aplicación de esta herramienta se opto por la validación hipótesis de manera individual, estos resultados se muestran en la Tabla 1.

Tabla 1: Resultados de validación de hipótesis (Departamento del Cesar vs Provincia de Sevilla)

\begin{tabular}{|c|c|c|c|c|c|c|c|c|}
\hline \multirow[b]{2}{*}{ Hipótesis } & \multirow{2}{*}{\multicolumn{2}{|c|}{ (Variables) }} & \multicolumn{3}{|c|}{ Departamento del Cesar } & \multicolumn{3}{|c|}{ Provincia de Sevilla [19]. } \\
\hline & & & Coeficiente & Covarianza & $\begin{array}{c}\text { Probabilidad } \\
\text { de }\end{array}$ & $\mathbf{r}$ & Relación & $\begin{array}{c}\text { Probabilidad } \\
\text { de }\end{array}$ \\
\hline 1 & $\begin{array}{l}\text { Tamaño de las } \\
\text { empresas con las } \\
\text { que se coopera }\end{array}$ & $\begin{array}{l}\text { La ubicación física de las empresas con las que se } \\
\text { establece algún acuerdo de cooperación. }\end{array}$ & 0,434 & + & SI & 0.795 & + & SI \\
\hline 2 & $\begin{array}{c}\text { La proximidad } \\
\text { física de las } \\
\text { empresas que } \\
\text { cooperan } \\
\end{array}$ & El desarrollo de innovaciones &.$^{\mathrm{a}}$ & $\mathbf{0}$ & No & $\begin{array}{c}- \\
0.356\end{array}$ & - & SI \\
\hline 3 & $\begin{array}{c}\text { EI tamaño de las } \\
\text { empresas }\end{array}$ & Edad de las empresa & 0,263 & + & No & 0.449 & + & SI \\
\hline 4 & $\begin{array}{c}\text { El tamaño de las } \\
\text { empresas }\end{array}$ & La certificación de sistemas de calidad & 0,402 & + & SI & 0.168 & + & NO \\
\hline 5 & $\begin{array}{c}\text { El tamaño de las } \\
\text { empresas }\end{array}$ & Innovación &.$^{\mathrm{a}}$ & $\mathbf{0}$ & No & 0.107 & $\mathbf{0}$ & SI \\
\hline 6 & Innovación & Ventas a Administraciones Públicas &.$^{\mathrm{a}}$ & + & No & 0.331 & + & SI \\
\hline 7 & $\begin{array}{c}\begin{array}{c}\text { La calidad en las } \\
\text { empresas }\end{array} \\
\end{array}$ & Ventas a administraciones públicas & 0,013 & + & No & 0.289 & + & SI \\
\hline 8 & $\begin{array}{c}\begin{array}{c}\text { La calidad en las } \\
\text { empresas }\end{array} \\
\end{array}$ & Innovación &.$^{\mathrm{a}}$ & $\mathbf{0}$ & No & 0.303 & + & SI \\
\hline 9 & $\begin{array}{c}\text { La necesidad de } \\
\text { financiación }\end{array}$ & La hostilidad del entorno & 0,337 & + & No & 0.153 & + & NO \\
\hline 10 & $\begin{array}{c}\text { La hostilidad del } \\
\text { entorno }\end{array}$ & La concentración de clientes. & $-0,147$ & + & No & $\begin{array}{c}- \\
0.098 \\
\end{array}$ & + & NO \\
\hline 11 & $\begin{array}{c}\text { La hostilidad o } \\
\text { rivalidad en el } \\
\text { entorno } \\
\end{array}$ & Innovación &.$^{\mathrm{a}}$ & $\mathbf{0}$ & No & $\begin{array}{c}- \\
0.003\end{array}$ & $\mathbf{0}$ & SI \\
\hline 12 & $\begin{array}{c}\text { La calidad en las } \\
\text { empresas }\end{array}$ & Promoción a directivos & $-0,046$ & + & No & 0.276 & + & NO \\
\hline 13 & $\begin{array}{c}\text { Las } \\
\text { organizaciones } \\
\text { con un control } \\
\text { formalizado } \\
\end{array}$ & Promoción a directivos & $\mathbf{0 , 4 3 3}$ & + & SI & 0.241 & + & NO \\
\hline 14 & $\begin{array}{c}\text { Las } \\
\text { organizaciones } \\
\text { más } \\
\text { concientizadas } \\
\text { socialmente } \\
\end{array}$ & problemas para reclutar personal & $-0,271$ & - & No & 0.26 & + & NO \\
\hline 15 & $\begin{array}{c}\begin{array}{c}\text { La calidad en las } \\
\text { empresas }\end{array} \\
\end{array}$ & problemas para reclutar personal & $-\mathbf{0 , 0 3 8}$ & - & No & 0.13 & - & NO \\
\hline 16 & $\begin{array}{c}\text { Relaciones } \\
\text { comercial con } \\
\text { administraciones } \\
\text { públicas } \\
\end{array}$ & apoyo de las administraciones públicas & $-0,191$ & - & No & 0.31 & + & SI \\
\hline
\end{tabular}

\section{PERFIL/MODELO EMPRESARIAL}

56 La Empresarialidad describe estructuras (variables contempladas) y nivel de calidad (valores o indicadores actuales) de las empresas en estudio, facilitando sintetizar su diagnostico, por ello se establecen perfiles empresariales que permiten definir de manera desagregada y estructurante las variables seleccionadas [21].

\section{PERFIL DE IDENTIFICACIÓN}

Se distingue una empresa con perfil de identificación, ubicada entre las dos principales ciudades del departamento (Valledupar y Aguachica), de gran tamaño de antigüedad entre 23 y 13 años, de carácter no familiar, sociedad anónima, no perteneciente a un grupo empresarial y con ubicación en una zona rural por razones de las instalaciones y el terreno disponible. La representación gráfica se observa en la figura 1 : 


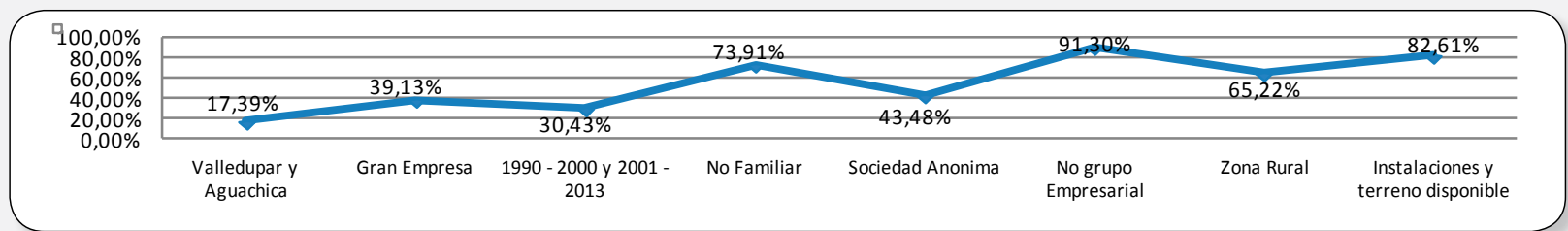

Figura 1: Perfil de Identificación

\section{PERFIL DE ORIENTACIÓN HACIA LA COMPETENCIA}

- Innovación de Productos o Servicios: se realizan innovación en los últimos y próximos tres años, enfocadas hacia productos modificados sustancialmente sobre los existentes en el pasado y en el futuro productos modificados levemente; El principal factor motivados de estas innovaciones es el incremento del mercado (o descubrimiento). La representación gráfica se observa en la figura 2:

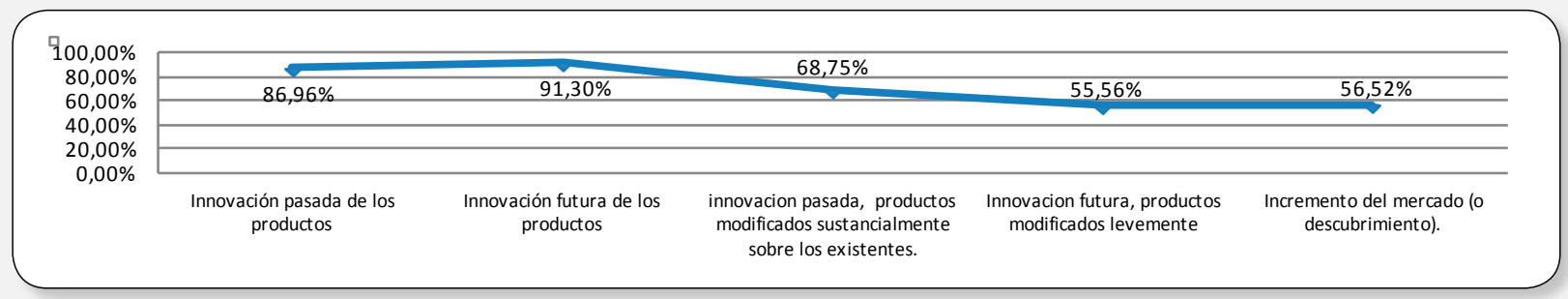

Figura 2: Perfil de innovación de productos o servicios

- Innovación de Procesos Productivos: Se afirma Innovación hace 3 años y tendencia a innovar en los próximos 3 años en los procesos productivos, enfocada hacia introducción de nuevos sistema de información y comunicación como innovación pasada e Introducción de nuevos equipos informáticos como innovación futura, buscando mantener el nivel actual de competitividad. La representación gráfica se observa en la figura 3:

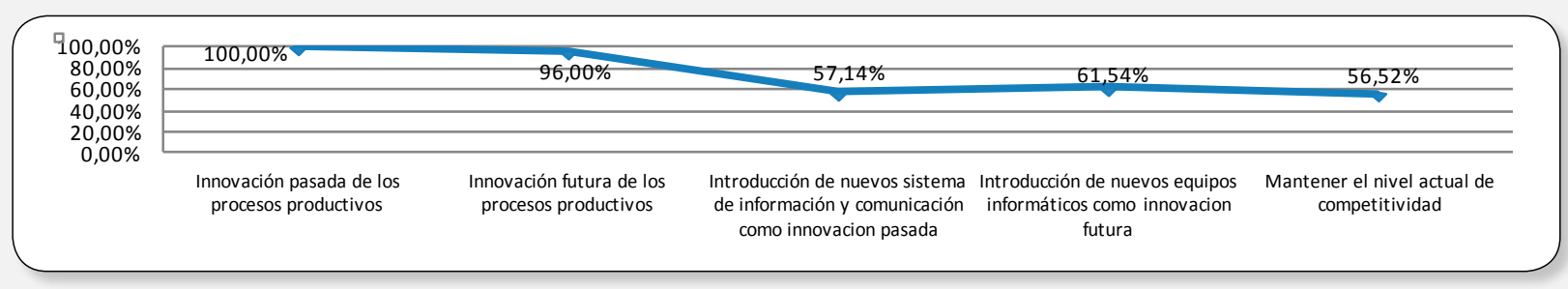

Figura 3: Perfil de innovación de procesos productivos

- Proceso Innovador y Principales Canales: como principales canales del proceso innovador, se destacan las asociaciones empresariales, proveedores de maquinaria, ferias y congresos. Además, se resaltan las personas inquietas y con iniciativas, y la conexión con los principales clientes como factor imprescindible en el proceso innovador. La representación gráfica se distingue en la figura 4 :

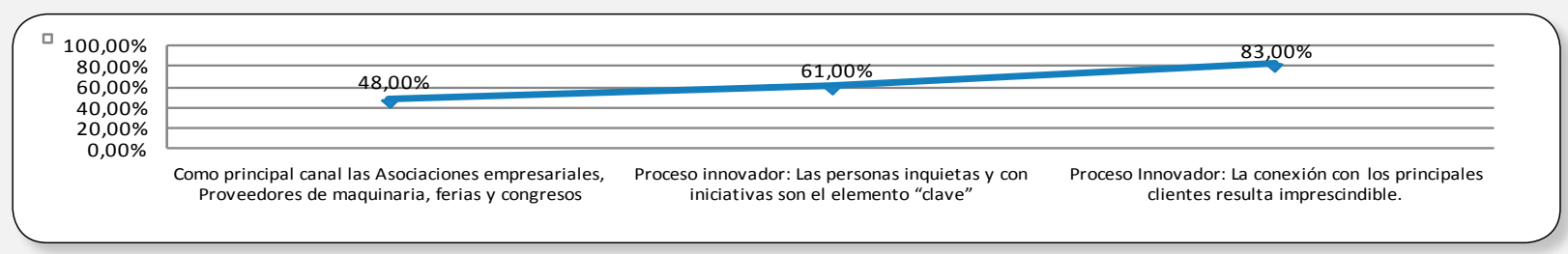

Figura 4: Perfil de proceso innovador y principales canales 
- Riesgos: Se destaca un grado de rivalidad fuerte, con tendencia de aumento, cambios rápidos y enfocados en la calidad. La representación gráfica corresponde a la figura 5:

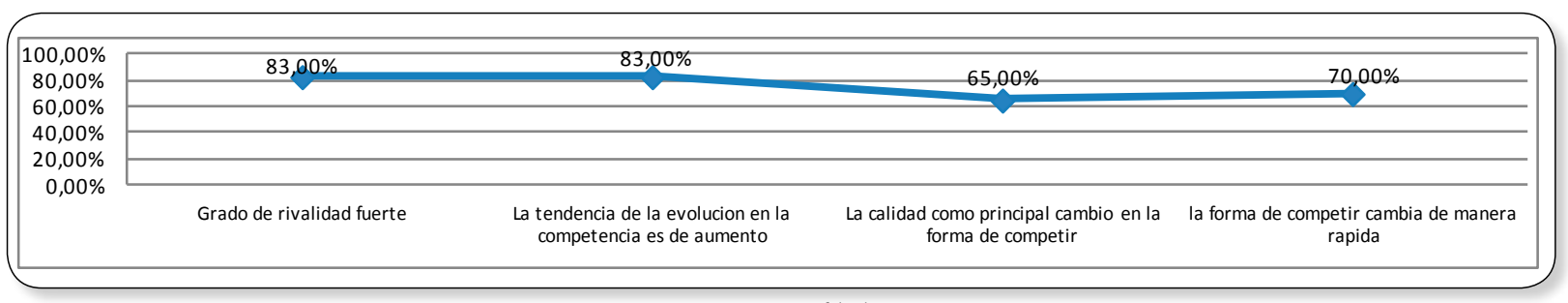

Figura 5: Perfil de riesgos

- Diferenciación: Finalizando la orientación hacia la competencia, en cuanto a la diferenciación, se identifica el diseño como factor muy importante para la empresa, al empresario como diseñador de los productos / servicios, la calidad como fundamental diferencia de los otros productos a los de la empresa, al igual que el principal factor de competencia; no comercializan marca propia, sin certificación de calidad y con medidas de satisfacción del cliente como principal acción para mejorar la calidad. La representación gráfica se distingue en la figura 6:

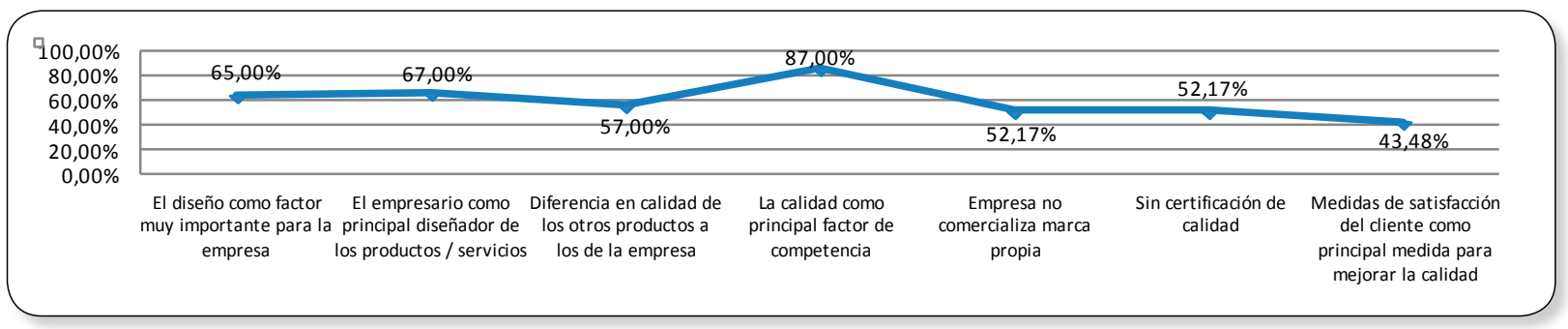

Figura 6: Perfil de diferenciación

\section{PERFIL DE ORIENTACIÓN HACIA EL CLIENTE}

Las ventas de las empresas se realizan en más del 50\% en el resto de la región, el principal y/o dos principales clientes absorben el total de estas, se presenta un crecimiento en los últimos tres años y con igual tendencia en los próximos tres años; afirman tener sistema postventa, sostienen que el diseño es un proceso exclusivamente interno el empresario, este ultimo como principal canal facilitador de relación con clientes extranjeros. La representación gráfica se puede ver en la figura 7:

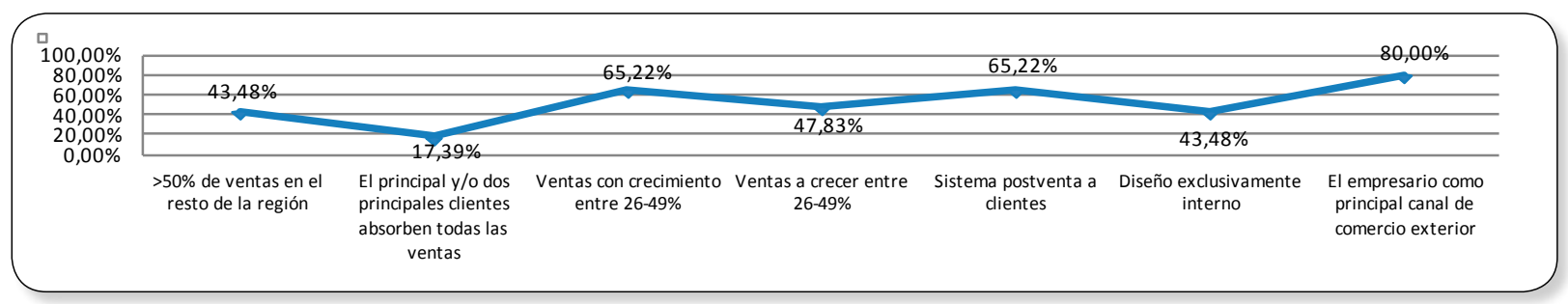

Figura 7: Perfil de orientación hacia el cliente

\section{PERFIL DE ORIENTACIÓN HACIA LOS RECURSOS HUMANOS}

- Planta de Personal: Se identifica que más de 200 empleados constituyen la planta de personal, mayor de 17.685.590.000 pesos aproximadamente equivalen los activos totales, menos del 10\% de los empleados es temporal; los jefes y directivos de áreas universitarios y los de nivel operativo 
ninguno profesional, además en ambos casos menos del 10\% son mujeres. En los últimos 3 años, el empleo ha crecido entre 26-49\%, se espera similar crecimiento en los próximos 3 años. Como mecanismo de reclutamiento de personal, se utilizan empresas de selección tanto para cargos directivos como operativos; el personal directivo procede del mismo departamento y el operativo del mismo municipio. En el reclutamiento del personal/ capacitaciones se presentan dificultades, la falta de formación necesaria es su principal factor. Sin embargo, las empresas que no tienen problemas en el reclutamiento del personal es debido a que el sector atrae mano de obra suficiente y el procedimiento que utilizan las empresas en la aprendizaje del personal es la capacitación en el puesto de trabajo. Su representación gráfica se observa en la figura 8:

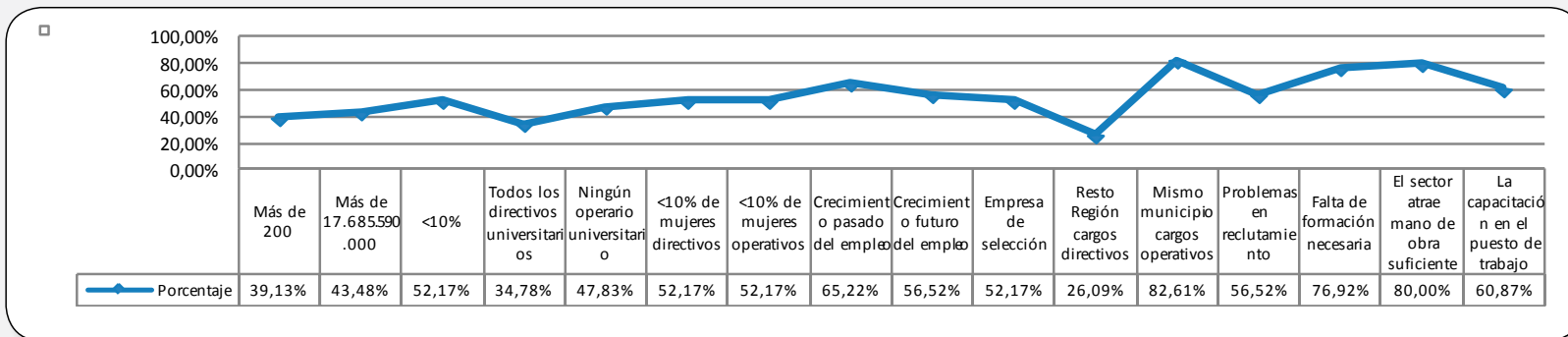

Figura 8: Perfil de planta de personal

- Dirección y Organización: Durante los proceso de selección de personal, se considera más importante la experiencia del candidato, el requisito prioritario exigido por el empleado son los beneficios salariales, los beneficios por objetivos alcanzados constituyen la mejor manera de motivar a un empleado, la experiencia y cualificación lograda son los criterios que se utilizan en mayor proporción para promocionar a los empleados. En el momento que surgen problemas en las tareas diarias habitualmente el individuo se dirige a su jefe inmediato. Lo anterior se representa gráficamente en la figura 9:

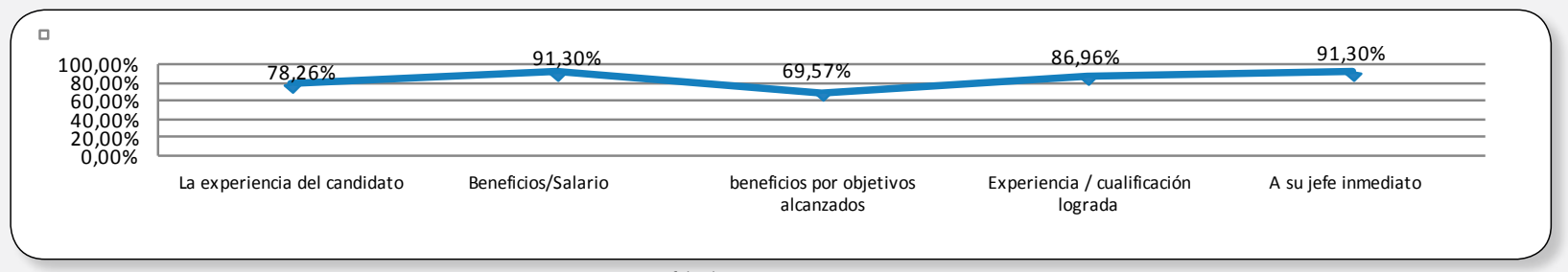

Figura 9: Perfil de Dirección y organización

\section{PERFIL DE ORIENTACIÓN HACIA EL ASOCIACIONISMO}

Las empresas tienden a no establecer acuerdos de colaboración, de cualquier tipo con otras empresas. Los que son realizados, son temporales de ámbito Investigativo, desarrollo y/o producción, con empresas pequeñas y medianas a nivel local y regional; Las empresas en mayor proporción no reciben ayuda por participar en acuerdos de colaboración, las recibidas son por parte de las administraciones local, departamental y/o nacional; además, no pertenecen a alguna asociación empresarial; sin embargo, las empresas que sí pertenecen a ellas les resulta de gran utilidad y su permanencia es de 5 años o más. Su representación gráfica se distingue en la figura 10: 


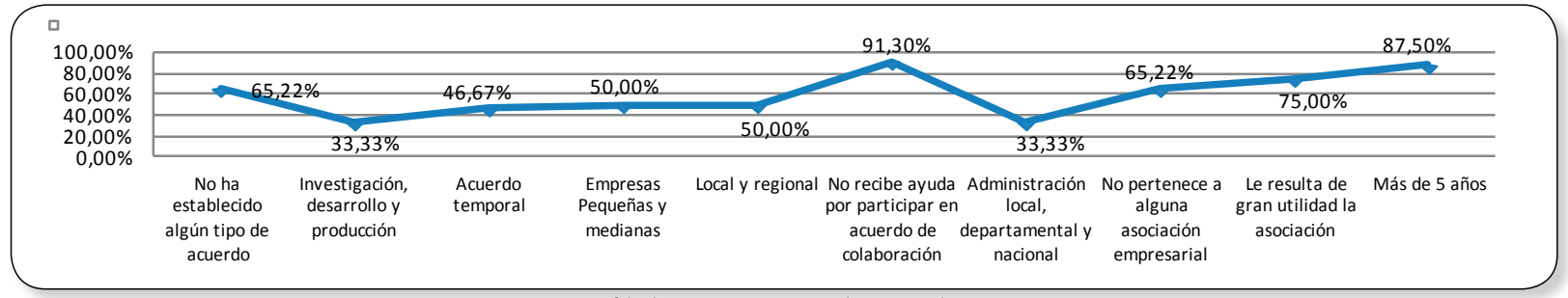

Figura 10: Perfil de orientación hacia el asociacionismo

\section{PERFIL DE ORIENTACIÓN HACIA LA ESFERA FINANCIERA}

Un solo socio posee más del 50\% del capital y la procedencia es del resto del país. Respecto a las políticas de autofinanciación y reparto de dividendos no es preciso tenerla definida. La financiación ajena, resulta fundamental tanto así que se facilita a corto y largo plazo. La representación gráfica se distingue en la figura 11:

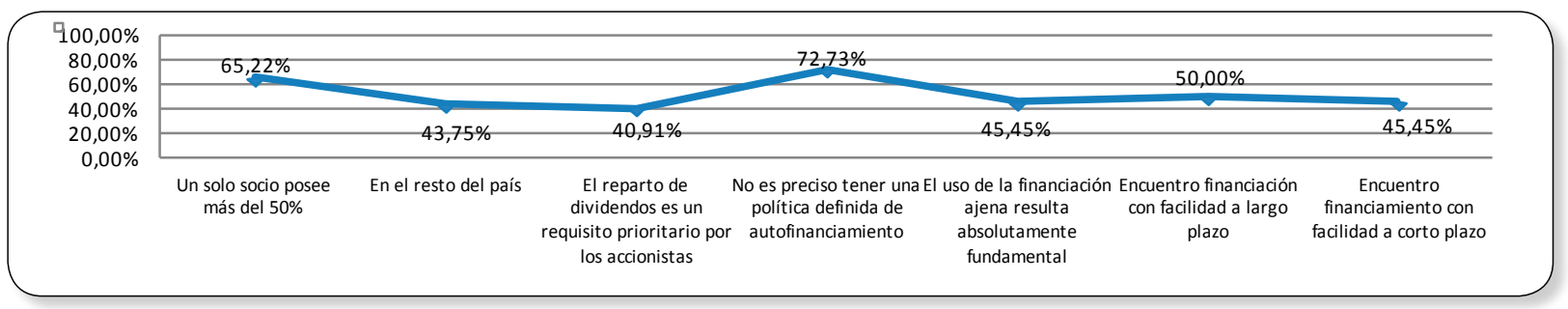

Figura 11: Perfil de orientación hacia la esfera financiera

\section{PERFIL DE ORIENTACIÓN HACIA LAS ADMINISTRACIONES PÚBLICAS}

En esta orientación, se destaca la ausencia de las administraciones públicas como clientes, debido a que no existe mercado para estas; en igualdad de condiciones, es preferible el cliente privado. Por último, respecto a la relación burocrática, algunas empresas consideran que no perciben colaboración ni comprensión por las administraciones públicas, pero otra parte sostiene que existe respaldado de parte de ellas en el desempeño de su actividad empresarial. Su representación gráfica se observa en la figura 12:

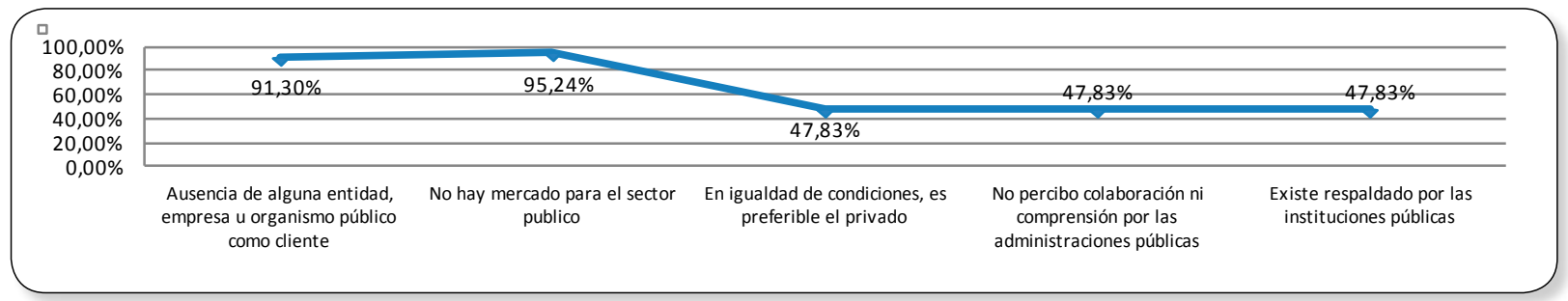

Figura 12: Perfil del mapa relacional de inquietudes empresariales

\section{PERFIL DE ORIENTACIÓN HACIA LA SOCIEDAD}

Se identifica la creación/mejora del empleo como un objetivo muy importante para el empresario, al igual de afirmar que el papel del empresario es fundamental para la sociedad. Por otro lado, se percibe una imagen positiva del empresario y cierto grado de aceptación del objetivo esencial de la empresa en contribuir a la creación de la riqueza nacional. Es representada por la figura 13: 


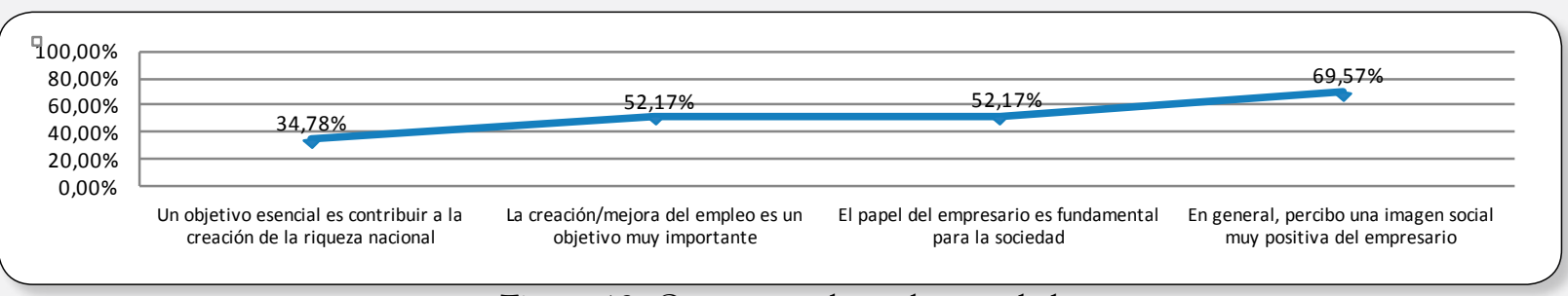

\section{PERFIL DE INQUIETUDES EMPRESARIALES}

En primer lugar se encuentra la materia prima, que en general, encierra problemas en su calidad, transporte y disponibilidad; en segundo la maquinaria, asociado a las necesidades de nuevas piezas que en el mercado Colombiano no se producen o comercializan, además de los requerimientos de tecnología especializada. Se representa por la figura 14:

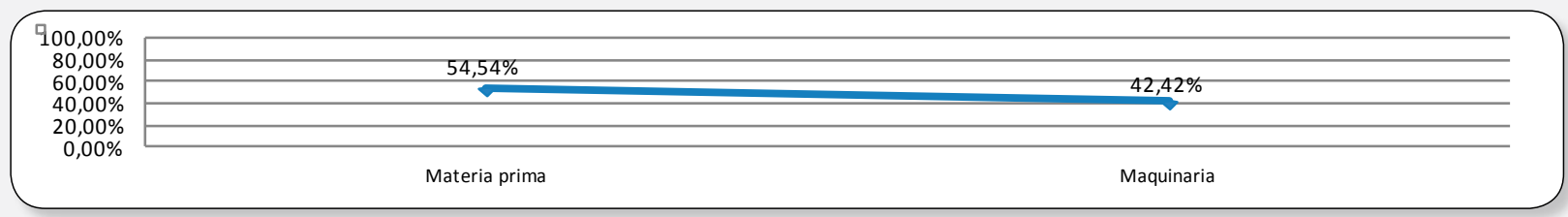

Figura 14: Perfil de inquietudes empresariales

\section{DISCUSIÓN Y CONCLUSIÓN}

De los resultados obtenidos, en el departamento del Cesar tres (3) de las hipótesis son validas y trece (13) se descartan, a diferencia de la investigación en Sevilla donde existen mayor relación entre las variables estudiadas (9 validas), debido a las diferencias microeconómicas, territoriales y cultura empresarial.

Entre las empresas agroindustriales del departamento del Cesar existe una gran relación entre el tamaño de las empresas con las que se coopera y la ubicación física de las empresas con las que se establece algún acuerdo de cooperación.

El tamaño de las empresas se relaciona significativamente con la certificación de los sistemas de calidad. Es decir, las empresas con mayor tamaño son las que tienden certificar sus sistemas de gestión de la calidad. Estos sistemas de gestión basados en normas nacionales e internacionales se han diseñado para cualquier tamaño de empresa, sin embargo su certificación lleva a mayores exigencias a las organizaciones que desean lograrla; por ello, se sugiere a las administraciones públicas, empresarios, federaciones u otras de interés, que implementen medidas para incentivar que las micros, pequeñas y medianas empresas (62,5\% de las empresas agroindustriales en el Cesar) implementen y certifiquen sus sistemas de gestión de la calidad, mejoraría la competitividad de las mismas, y mejoraría su expectativa ante el cumplimiento de este requisito indispensable en los mercados nacionales e internacionales, más aun cuando los empresarios encuestados afirman que la calidad es el factor principal de diferenciación de los otros productos a los de su empresa, al igual que el fundamental en cuanto a competencia. La última relación validada en las hipótesis, establece que las empresas del departamento del Cesar con un control formalizado, son las que promocionan a sus directivos según su experiencia y capacitación 
profesional, en detrimento de la creatividad. Aunque resulte conveniente realizar este tipo de promoción en donde se promueve una cultura en que la experiencia y cualificación representan el criterio fundamental para mejorar la motivación de los empleados, se está afectando el incentivo de la innovación en las empresas, porque el control formalizado limita el establecimiento de nuevos métodos y mejoramiento continuo de los procesos como factores a considerar en la promoción.

Por ello, se recomienda el establecimiento de medidas basadas no solo en experiencia y nivel profesional, sino en la proposición de nuevas ideas, para así ser coherentes con la afirmando de los empresarios al identificar a los empleados "inquietos y con iniciativas" como un factor clave en el proceso innovador.

Los perfiles propuestos, permiten identificar en primera instancia dispersión territorial media por parte de las empresas al situarse en 11 municipios de los 25 del departamento del Cesar, lo que podría estar asociado al grado de desarrollo económico que tienen estos municipios y al bajo nivel industrial de las empresas. Se denota perdida del aprovechamiento de los recursos en las zonas rurales y cabeceras municipales de los mismos; además se observa que la cultura organizacional de estas empresas se ha impuesto sobre la cultura familiar. También, la cultura emprendedora en el departamento del Cesar se ha afectado en el siglo XXI y las empresas han alcanzado madurez al iniciar operaciones en los años $60^{\mathrm{a}}$ y $70^{\mathrm{a}}$.

Por consiguiente, en el departamento del Cesar, la tendencia emprendedora tiende a disminuir, se hace necesario tomar medidas para incentivarlo. Por otra parte, las líneas industriales del Departamento no están en el máximo aprovechamiento con los productos primarios que abundan en la zona, muestra de ello es la experiencia de empresas como Frutas Chimichagua ubicada en el municipio que lleva su mismo nombre, esta desaparece a causa de falta de inversión, donde cosechas de mango no son priorizadas para la industrialización en este municipio. Se identificó además, la ausencia de zonas y/o parques industriales en el departamento, debido a la dispersión que presentan las empresas en su funcionamiento. Lo anterior, resulta desventajoso para la zona (departamento del Cesar) debido a que esta dispersión desestimula la generación y ubicación de nuevas empresas en el territorio.

Es de destacar los resultados presentes en el perfil de competencia y clientes, ya que estos demuestran el rezago del subsector agroindustrial, primeramente afirman en su mayoría un alto potencial innovador, pero con bajo niveles de sistemas de gestión de la calidad certificados, aunque ambas variables se enfocan a la satisfacción del cliente, los productos no sobrepasan los clientes de la región y el poder de decisión es significativamente bajo. Es aun más inquietante que los empresarios identifiquen como principal factor de competencia la calidad, pero la certificación no es priorizada.

Tanto a nivel nacional (mercados altamente competitivos) e internacionales como el caso de la Unión Europea, la calidad alimentaria y de productos son requisitos excluyentes.

A pesar de identificarse un aumento en la contratación de empleados en las empresas encuestadas, se pierde el potencial de mano de obra femenina al representar menos del $10 \%$. La experiencia se ha establecido como principal factor de selección en las empresas, lo que genera un desestimulo de la sociedad, prefiriendo vinculación laboral temprana que adquirir mayor formación.

El nivel de asociacionismo y establecimiento de convenios con otras empresas y entidades/instituciones/organizaciones es considerablemente bajo en las empresas estudiadas, los resultados son nulos aun mas a nivel internacional; los procesos de globalización de las empresas estudias han sido comunes, tanto en acuerdos comerciales como en otros tipos de vínculos presentan rezago. La sugerencia es dirigida a las instituciones encargadas de los procesos de internacionalización, los empresarios, 
asociaciones gremiales, entre otros; debido a que el potencial y necesidades identificadas en los perfiles propuestos podrían maximizados y satisfecho respectivamente con transferencia tecnológica, facilitándose el proceso en articulación con la academia.

La facilidad de financiación a corto y largo plazo como factor fundamental para las empresas encuestadas, permite señalar que la inversión no representa en mayor proporción la barrera para la operación y mejoramiento de las empresas agroindustriales en el departamento del Cesar.

En cuanto la relación de las empresas con las administraciones públicas, se evidencia una desconexión entre estos actores, para el establecimiento de políticas económicas la utilización de herramientas para diagnosticar según variables como la implementada en la actual investigación, facilitan la identificación de debilidades, fortalezas, amenazas y oportunidades para las mismas, y así priorizar políticas públicas de índole económico y social.

A pesar de que se identifica una imagen positiva y orientación hacia la sociedad por parte del empresario, además de resaltar la vinculación de personal oriundo de la zona donde se localizan las empresas, se recomienda a la implementación de diagnostico de las comunidades vecinas, permitiendo focalizar sus estrategias de responsabilidad social, no solo con orientación a los Stakeholders internos, sino además a los grupos de interés externos. La presente investigación identifica la necesidad de vinculación de las mujeres, ya que como se menciono anteriormente su vinculación laboral está por debajo del 10\%

Por otra parte, entre las empresas del departamento del Cesar se evidencian dos principales problemas: la materia prima y la maquinaria; en cuanto al primer elemento se hace necesario ejercer un mayor control sobre los proveedores, por medio de su vinculación en la búsqueda de soluciones en lo referente a calidad, transporte y disponibilidad. Además, se sugiere a las empresas la implementación de sistemas de integración hacia atrás para ejercer mayor seguimiento y control a su cadena de valor, como también puesta en marcha de auditorías internas de sus proveedores. Por otra parte, las empresas deben garantizar que la maquinaria adquirida les permita manejar stop de piezas, eficiencia en los sistemas postventa, respuestas ante pedidos, entre otros; por medio de la respectiva evaluación.

Para finalizar, con la validación de las hipótesis, comparación con Sevilla y el establecimiento de los perfiles empresariales, se facilitó la descripción y comprensión del subsector agroindustrial del departamento del Cesar.

\section{AGRADECIMIENTOS}

El autor expresa sus agradecimientos primeramente a Dios por la sabiduría brindada, al programa "Jóvenes Investigadores e Innovadores" de COLCIENCIAS y la Universidad de Santander (UDES) por el apoyo.

\section{BIBLIOGRAFÍA}

[1]. GOBERNACIÓN DEL CESAR, Plan de Desarrollo para el Departamento del Cesar 2012 - 2015. 2012

[2]. DANE: Informe de Coyuntura Económica Regional. ICER. Cesar. I Semestre de 2006.

[3]. O’KEAN, J. M. La Función Empresarial ante la Nueva Economía. http://www. juntadeandalucia.es/economiayhacienda /web/economia/estudios/bea/descarga/TOMO_33/ BEA33_186.pdf. (Consultado el $\overline{30}$ de agosto de 2012)

[4]. BONET, J. Minería y Desarrollo Económico en el Cesar. Documentos de Trabajo sobre Economía Regional. Cen-
Enero - Junio 2014

ISSN 0122-820X

PP: 50 - 65 
No. 1

[5]. VILORIA, J.La Ganadería Bovina en las Llanuras del Caribe Colombiano. Documentos de Trabajo sobre Economía Regional. Centro de estudios Económicos Regionales CEER. Banco de la República. Cartagena. 2003

[6]. GAMARRA, J. La Economía del Cesar después del Algodón. Documentos de Trabajo sobre Economía Regional. Centro de estudios Económicos Regionales CEER. Banco de la República. Cartagena. 2005.

[7]. AgUilERA, M. Aguachica: Centro Agroindustrial del Cesar. Documentos de Trabajo sobre Economía Regional. Centro de estudios Económicos Regionales CEER. Banco de la República. Cartagena. 2004

[8]. ZAPATA B. En la Construcción de la Historia Empresarial de Valledupar: Una Mirada a Tres Décadas, 1950-1980. 2013

[9]. TAMAYO Y TAMAYO, Mario. El proceso de la Investigación Científica, Ediciones Limusa, 2009.

[10]. BRIONES, Guillermo. Metodología de la Investigación Cuantitativa en las Ciencias Sociales. Instituto colombiano para el fomento de la educación superior, ICFES. Especialización en teoría, métodos y técnicas de investigación social. Composición electrónica: A R FO Editores e Impresores Ltda. Diciembre de 2002. Pag. 104 - 175.

[11]. LÓPEZ GIL, Efraín. El proceso de investigación. Armenia: Universidad del Quindío, 1995, p.70.
[12]. PORTER, M.E. La Ventaja Competitiva de las Naciones. Barcelona: Plaza \& Janés, 1991.1056 pp

[13]. LEONTIADES, M. Strategy and Reality: A Challenge for Business Policy". Academy of Management Review, Vol. 4, no 2, 1979, pp. 275- 279.

[14]. LOBER, D.J. y BAILEY, M. Organizational Strategy, Managerial DecisionMaking, and Market Based Environmental Policies: Utility Company Bidding Behavior in the Sulfur Dioxide Allowance Trading Auctions. Managerial and Decision Economics, Vol. 18, no 6 , 1997, pp. 471-499.

[15]. LERMA, H. Metodología de la Investigación. Bogotá D.C.: ECOE Ediciones. 2001.

[16]. DEPARTAMENTO NACIONAL DE PLANEACIÓN (DNP). Visión Cesar Caribe 2032: Un departamento en crecimiento generando bienestar. ISSN: 2256-1854. 2011.

[17]. FEDEPALMA. Minianuario Estadístico de Palma 2013. Pagina web: http:// fedepalma.portalpalmero.com/bigdata/ fedepalma/ pdf/minianuarioestadistico_2013.pdf. (Consultado el 15 de febrero de 2013)

[18]. GUZMÁN K. La industria láctea en Valledupar. Documentos de Trabajo sobre Economía Regional. Centro de estudios Económicos Regionales CEER. Banco de la República. Cartagena. 2013

[19]. TAMAYO, J., GAMERO, J., MARTÍNEZ, J.A. "Exploración del entorno empresarial de Sevilla con un mapa de relación bidimensional". Cuadernos de Estudios Empresariales 2006, vol. 16. 135-155. 
[20]. UNIVERSIDAD DE SEVILEnero - Junio 2014 LA. http://personal.us.es/vararey/ adatos2/correlacion.pdf (Consultado el ISSN 0122-820X 14 de Marzo de 2013)

[21]. SANTOS CUMPLIDO, J. (2004). Convergencia, Desarrollo y Empresarialidad en el Proceso de Globalización Económica. Revista de Economía Mundial. № 10/11. Pág. \{171-202\}. 